\title{
INTENTOS DE SUICIDIO POR INTOXICACIÓN CON SUSTANCIAS QUÍMICAS EN COLOMBIA. 2007 - 2013
}

\section{SUICIDE ATTEMPTS BY CHEMICALS POISONING IN COLOMBIA. 2007 - 2013}

\author{
TITULO CORTO: INTENTOS DE SUICIDIO POR INTOXICACIÓN CON \\ SUSTANCIAS QUÍMICAS
}

Viviana Calderón-Ramírez ${ }^{1}$, Adalgisa Alcocer-Olaciregui ${ }^{2}$, Rusvelt Vargas-Moranth ${ }^{3}$

Recibido en abril 01 de 2016

Aceptado en agosto 18 de 2016

Publicado en línea en mayo 15 de 2017

\section{Resumen}

El objetivo de este trabajo fue establecer el perfil epidemiológico de los intentos de suicidio por sustancias químicas en Colombia durante el período 2007-2013. Para ello se llevó a cabo un estudio descriptivo transversal con datos del sistema de vigilancia en salud pública SIVIGILA por intoxicaciones con sustancias químicas 2007-2013. Se encontró que el $55 \%$ de los casos en los siete años de estudio corresponde al género femenino. El $73 \%$ de casos se presenta desde los 10 hasta los 29 años, con mayor concentración (31\%) en el grupo de 15 a 19 años. Por pertenencia étnica, el Negro, Mulato y Afro Colombiano muestran un alto número de casos desde 2007 a 2009; mientras que los indígenas muestran una tendencia ascendente desde el 2011 hasta 2013. La mayoría de las personas que intentan suicidarse son solteros (58\%), tienen un nivel de escolaridad secundaria (50\%), y viven en una cabecera municipal. Aproximadamente el 0,05\% de las mujeres que intentaron quitarse la vida estaban en estado de embarazo. En conclusión, se encontró una importante incidencia de intentos de suicidio con sustancias químicas que debe ser analizada teniendo en cuenta las características socioculturales de las regiones y los grupos poblacionales que componen la nación colombiana.

Palabras clave: Suicidio; intento; químicos.

\section{Abstract}

The objective was to set the epidemiological profile of suicide attempts by chemical substances in Colombia during the period 2007-2013. It was carried out a descriptive cross sectional study. Data were obtained from Public Health Surveillance system (SIVIGILA) about poisoning chemicals 2007-2013. It was found that $55 \%$ of cases in 7 years of

1. Psicóloga, Magister en Salud Pública. Grupo de investigación en epidemiología aplicada, Instituto Nacional de Salud. Colombia. Correo: viviadcalderon@gmail.com 2. Ingeniera de sistemas. Magister en Epidemiologia. Universidad Metropolitana de Barranquilla. Colombia. Correo: aalcocer@unimetro.edu.co

3. Médico, Magíster en Salud Pública. Universidad Metropolitana de Barranquilla. Estudiante doctorado en Ciencias Biomédicas-Economía de la Salud. Universidad de Cartagena. Colombia. Correo: rvargas@unimetro.edu.co 
study corresponds to the female gender. $73 \%$ of cases occur from 10 to 29 years, with the highest concentration (31\%) in the group of 15 to 19. By ethnicity the Black, Afro Colombian Mulato and shows a high number of cases from 2007 to 2009 and indigenous show an upward trend from 2011 to 2013. Most people who attempt suicide are single (58\%) have a high school education $(50 \%)$ and live in municipal head. Approximately $0.05 \%$ of the women who tried to kill themselves, were in a state of pregnancy. In conclusion, a significant incidence of suicide attempts was found with chemicals, and for analysis must take into account the socio-cultural characteristics of the regions and population groups that make up the Colombian nation

Keywords: Suicide; intent; chemicals.

\section{INTRODUCCIÓN}

$\mathrm{E}^{\mathrm{n}}$ n los últimos 50 años las tasas de suicidio han aumentado en un $60 \%$ a nivel mundial ${ }^{1}$. En Colombia, el suicidio es la cuarta forma de violencia con una tasa de mortalidad de 4,3 por cada 100.000 habitantes. Cada año, por cada suicidio cometido se presentan muchos más intentos y, si se observan por mecanismo, las reincidencias de notificación por intoxicación son de hasta cuatro notificaciones en dos años, de las cuales el $73 \%$ corresponde a intento de suicidio ${ }^{2}$.

Estas reincidencias se explican a partir de la relación que hay entre el suicidio y el intento de suicidio con algún grado de depresión ${ }^{3}$. Lo que sugiere, simultáneamente, que los seis primeros meses e, incluso, el primer año después del intento, el riesgo aumenta de 20 a 30 veces $^{4}$, razón por la cual se hace necesario brindar intervención inmediata.

En los países occidentales, entre el 15\% y el 20\% de las emergencias por intento de suicidio son causadas por intoxicaciones químicas 5 . En este sentido, se advierte que alrededor del $75 \%$ de los métodos de suicidio corresponden a intoxicaciones químicas, y que estas se presentan en un $24,7 \%$ de los casos consumados ${ }^{6}$, siendo los pesticidas el método más común de auto-envenenamiento en los países en desarrollo ${ }^{7}$. En Colombia, el último boletín epidemiológico del año 2015 mostró el acumulado anual para el evento "Intencionalidad suicida por intoxicaciones": de los 14,477 casos que se presentaron, el $53,5 \%$ de los mismos correspondió a intoxicaciones por medicamentos y el $34,4 \%$ a intoxicaciones por plaguicidas. Alrededor de una tercera parte de los casos se dieron en jóvenes entre los 15 y los 19 años, mientras que el 19,7\% se reportaron en adultos jóvenes entre los 20 y los 24 años ${ }^{8}$.

Según el Instituto Nacional de Salud", el "intento de suicidio" se define como: "conducta potencialmente lesiva auto-infligida y sin resultado fatal, para la que existe evidencia, implícita o explícita, de intencionalidad de provocarse la muerte. Dicha conducta puede provocar o no lesiones, independientemente de la letalidad del método".

Los suicidios son evitables y, para que las respuestas a nivel global sean eficaces, se necesita una estrategia integral multisectorial de prevención con especial atención a los grupos de riesgo o de mayor vulnerabilidad identificados a partir de los datos recolectados en los sistemas de información, los datos científicos e investigaciones, tal como lo sugiere el plan de salud mental 2013-2020 propuesto por la OMS para los países adscritos, entre ellos Colombia ${ }^{10}$.

Hasta antes del año $2015^{9}$ no existía en el país un protocolo de vigilancia epidemiológica para los intentos de suicidio por intoxicación con sustancias químicas y por otros medios, a pesar de que los intentos previos de suicidio son el predictor más fuerte de un posible intento de suicidio en el futuro ${ }^{11}$, aumentando el riesgo de 20 a 30 veces $^{12}$ durante los primeros seis meses e, incluso, durante el primer año después del intento.

Por lo anterior, se hace necesario desarrollar investigaciones que sean el insumo para el establecimiento de estrategias que permitan hacer una detección temprana y una intervención oportuna con el fin de invertir, detener o, por lo menos, retardar el progreso de las causas de base que promueven el intento de suicidio, prevenir la consumación 
del evento y, a su vez, evaluar las acciones o programas que ya se están implementando como la vigilancia de intoxicaciones ${ }^{13}$.

Debido a ello, el objetivo del presente trabajo es describir el perfil epidemiológico de los intentos de suicidio por intoxicación con sustancias químicas en Colombia durante el período 2007-2013, teniendo en cuenta que, según el Instituto Nacional de Salud, la mayoría de defunciones notificadas por intoxicaciones con sustancias químicas durante los años 2011, 2012 y 2013 están asociadas principalmente a intoxicaciones de tipo intencional suicida por plaguicidas y por otras sustancias químicas ${ }^{14}$.

\section{MATERIALES Y MÉTODOS}

Estudio de tipo descriptivo transversal. La fuente de información utilizada fue secundaria; los datos se tomaron del sistema de vigilancia en salud pública SIVIGILA 20072013 del evento de intoxicaciones por sustancia química con intencionalidad suicida en Colombia, Instituto Nacional de Salud.

Se llevó a cabo un censo donde se incluyeron todos los casos registrados en el sistema de vigilancia de salud pública SIVIGILA de intoxicación por sustancias, desde el año 2007 hasta el 2013, que cumplieron con los criterios de selección.

\section{Criterios de inclusión}

Casos notificados como exposición a intoxicación por sustancia química con intencionalidad suicida y todos aquellos clasificados como otro que tuviese comentario de intención suicida en la base de datos del sistema de vigilancia en salud pública SIVIGILA de intoxicación por sustancias químicas entre los años 2007 y 2013. Los tipos de sustancias incluidos debían corresponder a los indicados por el protocolo de Intoxicaciones Químicas del INS ${ }^{14}$ :

Plaguicidas, medicamentos, metanol, metales pesados (mercurio, plomo, cromo), solventes (hidrocarburos alifáticos, hidrocarburos aromáticos, hidrocarburos halogenados, alcoholes, cetonas, éteres, ésteres y derivados del glicol), gases (asfixiantes e irritantes), otras sustancias químicas (ácidos, álcalis, agroquímicos, cosméticos, cianuro, fosforo blanco, pegantes, plantas y hongos tóxicos, y productos de limpieza del hogar), gases, sustancias psicoactivas (etanol, cocaína, marihuana, heroína, escopolamina, anfetaminas, metanfetaminas, metilendioximetanfetamina, lsd, hongos psilocybecubensis y otros, gama hidroxibutirato y nicotina).

\section{Criterios de exclusión}

Aquellos casos con otro tipo de exposición a intoxicación por sustancia química diferente al de intencionalidad suicida y aquellos clasificados como otros con comentario diferente al de intencionalidad suicida en la base de datos del sistema de vigilancia en salud pública SIVIGILA de intoxicación por sustancias químicas 2007 a 2013.

La información fue procesada en Microsoft Excel 2016 ${ }^{\circledR}{ }^{\circledR}$ y en Epiinfo $7^{\circledR}$. Se establece el número de casos registrados con intencionalidad suicida y se describen las características de persona y lugar (sexo, edad, nivel educativo, estado civil, pertenencia étnica, región geográfica).

\section{Declaración sobre aspectos éticos}

Se hizo solicitud oficial al Instituto Nacional de Salud (INS) de la base de datos del SIVIGILA referente a eventos de intoxicación con sustancias químicas 20072015, con fines investigativos. Esta fue entregada con compromiso de confidencialidad, sujeta a derechos de propiedad intelectual y anonimizada para garantizar la confidencialidad de los datos de identidad.

A pesar de que la fuente de información es secundaria y no se requiere contacto directo con el usuario del servicio de atención, se tuvo en cuenta la resolución 8430 de 1993 de investigación en salud, por la cual se establecen las normas científicas, técnicas y administrativas bajo el marco de investigación sin riesgo, así como la Declaración de Helsinki.

\section{RESULTADOS}

Se revisaron 32.097 casos de intento de suicidio por sustancias químicas, correspondientes a los años 2007 a 2013. Se encontró que el comportamiento del evento es heterogéneo cada año; sin embargo, se identificaron elementos notorios: por ejemplo, que en el rango de 15 a14 y de 20 a 24 años se concentra el mayor número de casos en los dos géneros, los cuales representan el $51 \%$ del total por grupos de edad en todo el período de los siete años (Figura 1). 

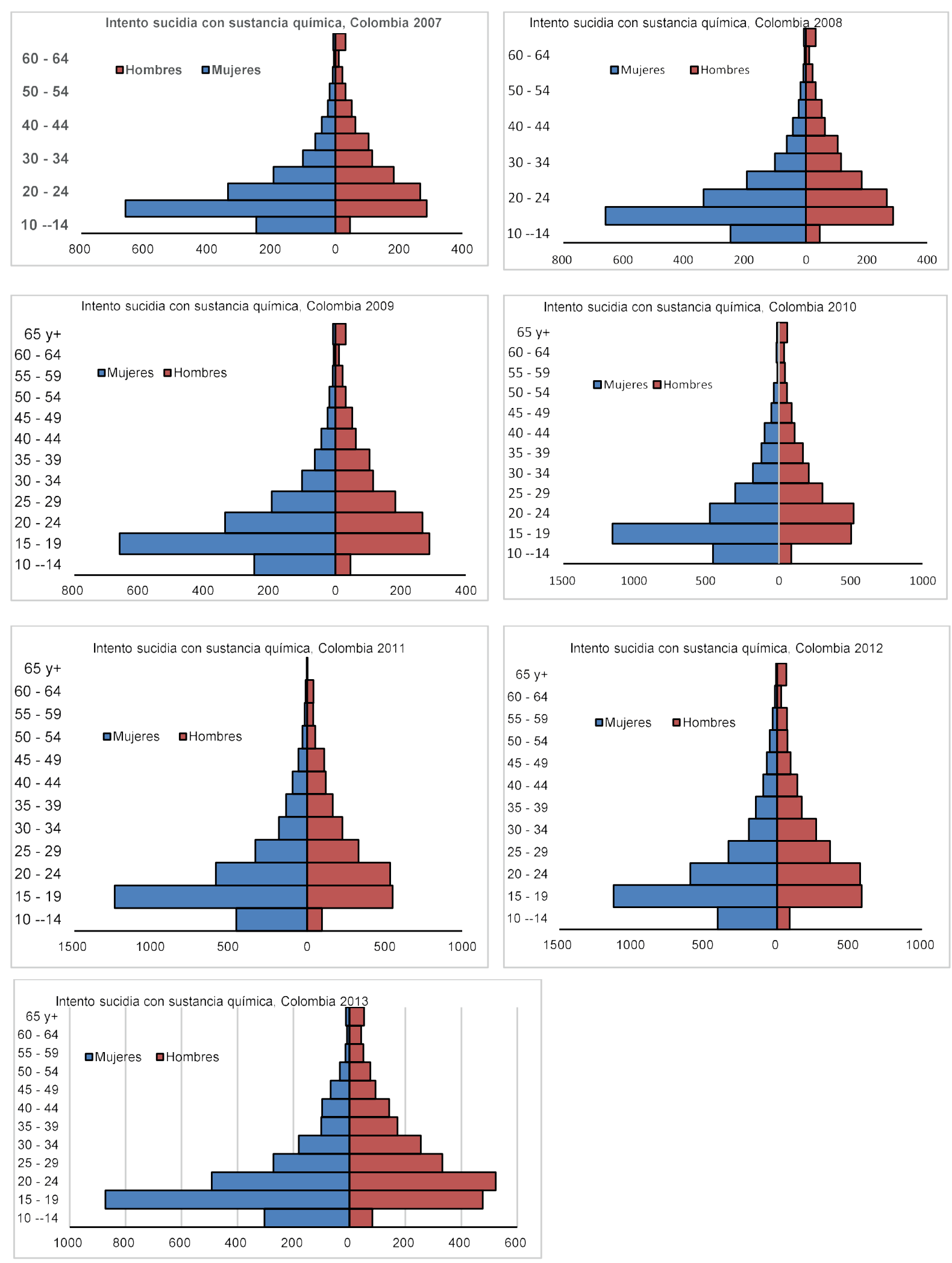

Figura 1. Comportamiento del intento de suicidio con sustancia química por edad y sexo, Colombia 2007-2013. Fuente: Elaboración de las autoras con datos SIVIGILA 2007-2013 
De los 32.097 casos estudiados, la mayoría (54,6\%) correspondían a mujeres. Al desagregar los casos por año se observó que, en todos los años, la mayor proporción de casos se presentó en mujeres, a excepción del año 2008 en el cual el porcentaje de hombres fue de $55,5 \%$ (Figura 2).
Por su parte, de acuerdo al sitio de residencia, las cabeceras presentan un porcentaje de intento de suicidio en incremento sostenido con respecto a otras zonas. Hasta el 2010, y como corolario, la proporción de casos ocurridos en otras zonas se va incrementando desde 2011 hasta 2013 (Tabla 1).

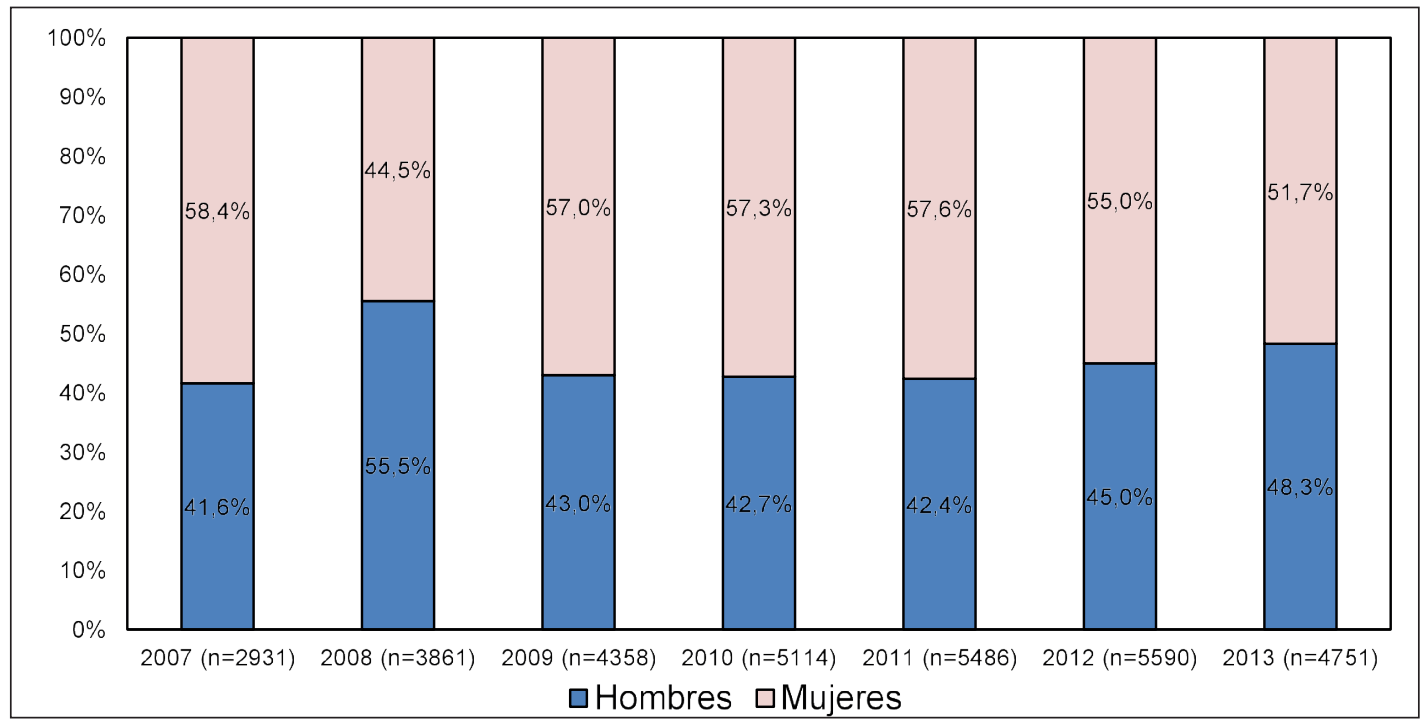

Figura 2. Distribución de intento de suicidio por sustancias químicas por género, Colombia 2007-2013.

Tabla 1. Distribución de intento de suicidio por sustancias químicas, por sitio de residencia, Colombia 2007-2013.

\begin{tabular}{|c|c|c|}
\hline \multirow{2}{*}{ Año } & \multicolumn{2}{|c|}{ Porcentaje (\%) de casos en } \\
\cline { 2 - 3 } & Cabecera & Resto \\
\hline $2007(n=2931)$ & 57,7 & 42,3 \\
\hline $2008(n=3861)$ & 57,0 & 43,0 \\
\hline $2009(n=4358)$ & 67,3 & 32,7 \\
\hline $2010(n=5114)$ & 67,7 & 32,3 \\
\hline $2011(n=5486)$ & 66,8 & 33,2 \\
\hline $2012(n=5590)$ & 63,7 & 36,3 \\
\hline $2013(n=4751)$ & 62,8 & 37,2 \\
\hline
\end{tabular}

Fuente: Elaboración de las autoras con datos SIVIGILA 2007-2013

En relación a la distribución de intentos de suicidio de acuerdo a la región del país, esta clasificación y agrupamiento de departamentos responde a criterios definidos por el Ministerio de Educación, los cuales se tuvieron en cuenta para el presente estudio así:

- Pacífica: Valle del Cauca, Nariño, Chocó y Cauca

- Caribe: Atlántica, Bolívar, Magdalena, La Guajira, Cesar, Córdoba, Sucre y San Andrés y Providencia.

- Andina: Antioquia, Boyacá, Cundinamarca, Caldas, Huila, Norte de Santander, Quindío, Risaralda, Santander, Tolima y el Distrito Capital.

- Amazonía: Caquetá, Putumayo, Guaviare, Amazonas, Guainía y Vaupés.

- Orinoquía: Meta, Casanare, Arauca y Vichada.

Se evidencia que, en todos los años estudiados, la mayor proporción de casos se encontró en la región andina con valores que van desde el 55,9\% en el 2007, hasta el 62,7\% en el 2010. En segundo lugar, la región pacífica aportó porcentajes de casos desde el 6, 2\% en el 2007 hasta el 12,6\% en el 2013. Y, en tercer lugar, la región caribe, con porcentajes que no superaron el $15 \%$ en cada uno de los años (Tabla 2). 
Tabla 2. Distribución de casos de intento de suicidio por sustancias químicas, según regiones geográficas y pertenencias étnicas. Colombia 2007-2013.

\begin{tabular}{|c|c|c|c|c|c|c|c|c|c|c|}
\hline \multirow{2}{*}{ Año } & \multicolumn{4}{|c|}{ Porcentaje (\%) de casos según región } & \multicolumn{4}{c|}{ Porcentaje (\%) de casos según etnia } \\
\cline { 2 - 12 } & Andina & Pacífica & Caribe & Orinoquía & Amazonía & Indígena & Rom & Raizal & Negro & Otros \\
\hline $2007(\mathrm{n}=2931)$ & 55,9 & 22,2 & 6,2 & 9,6 & 5,9 & 4,8 & 0,3 & 0,1 & 16,3 & 78,4 \\
\hline $2008(\mathrm{n}=3861)$ & 57,0 & 23,9 & 6,5 & 7,9 & 4,5 & 3,7 & 0,3 & 0,6 & 10,6 & 84,7 \\
\hline $2009(\mathrm{n}=4358)$ & 59,2 & 21,3 & 7,3 & 7,3 & 4,8 & 3,0 & 0,4 & 0,4 & 8,7 & 87,5 \\
\hline $2010(\mathrm{n}=5114)$ & 62,7 & 20,0 & 6,7 & 7,0 & 3,4 & 3,4 & 0,2 & 1,0 & 5,0 & 90,5 \\
\hline $2011(\mathrm{n}=5486)$ & 60,7 & 17,9 & 8,4 & 8,1 & 4,7 & 3,0 & 0,1 & 0,1 & 4,8 & 91,9 \\
\hline $2012(\mathrm{n}=5590)$ & 58,8 & 18,7 & 10,3 & 7,2 & 4,9 & 3,5 & 0,1 & 0,1 & 4,3 & 92,0 \\
\hline $2013(\mathrm{n}=4751)$ & 56,7 & 20,2 & 12,6 & 5,3 & 5,1 & 4,9 & 0,0 & 0,1 & 3,6 & 91,4 \\
\hline
\end{tabular}

Fuente: Elaboración de las autoras con datos SIVIGILA 2007-2013

En lo correspondiente a la pertenencia étnica (Tabla 2), se identifica que el grupo "otros" es el que concentra mayor número de casos durante los siete años estudiados, con un comportamiento ascendente desde el 2007 hasta el 2012, y una leve reducción en el 2013. El grupo que representa Negro, Mulato y Afrocolombiano muestra un alto número de casos durante 2007, 2008 y 2009, y en los años posteriores un descenso progresivo.
Por su parte, el grupo indígena presenta una tendencia ascendente con mayor número de casos desde el 2011 hasta el 2013.

Así mismo, entre todos los grupos étnicos el más afectado es el de Negro, Mulato y Afrocolombiano representando el $7 \%$ del total de los siete años de estudio, seguido del grupo indígena con el $4 \%$ (Figura 3).

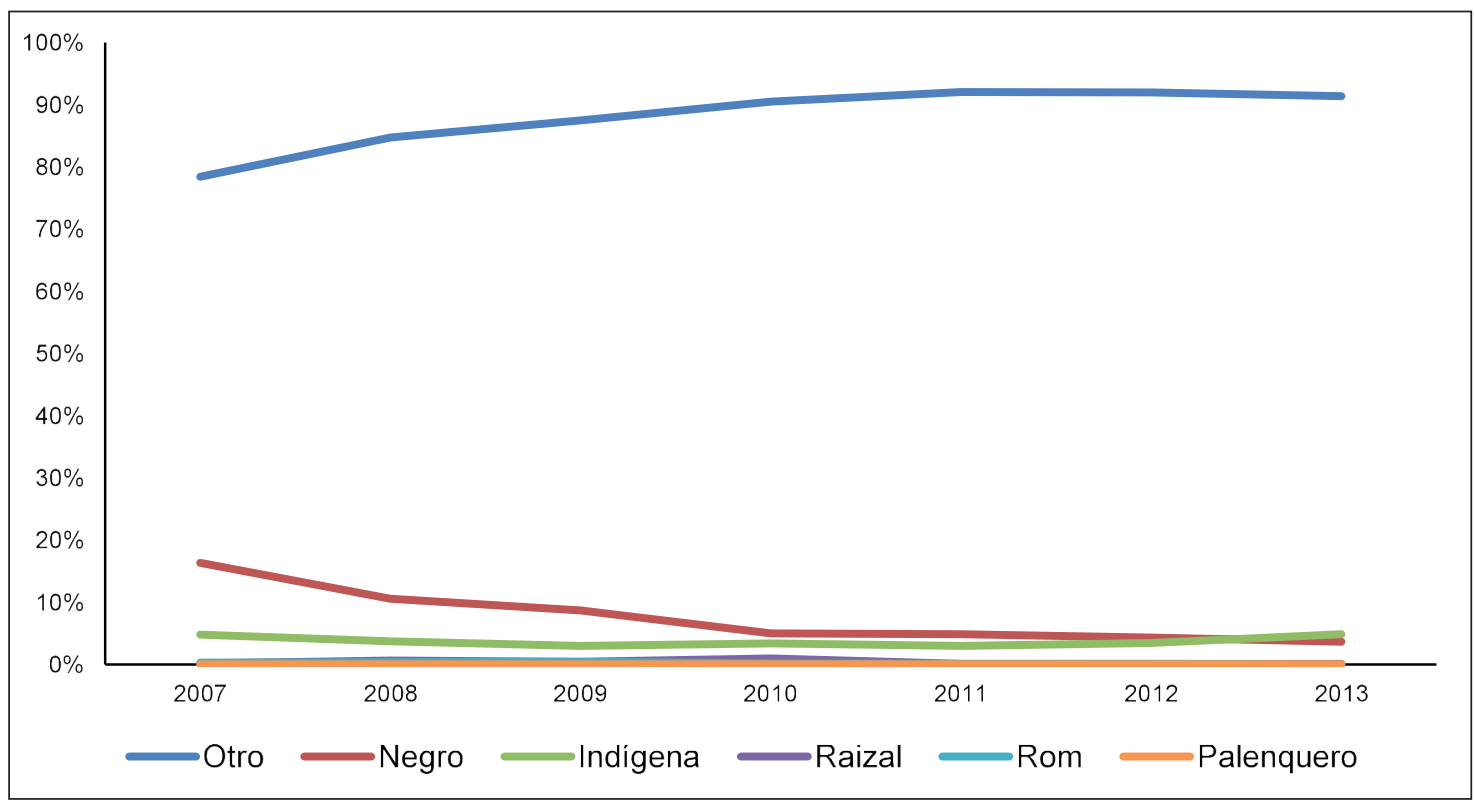

Figura 3. Intentos de Suicidio por sustancias químicas, según pertenencia étnica. Colombia 2007-2013. 
Por otro lado, en cuanto al nivel de escolaridad, el 6,4\% de los casos se presentan en personas con ningún nivel de estudios, el $40 \%$ en personas con nivel de escolaridad primaria, el $50 \%$ en personas con nivel de secundaria, y en menor proporción en personas con nivel de estudios universitarios. En los siete años observados se identifica un descenso en el año 2013, presentándose 324 casos menos que el año inmediatamente anterior (2012) en las personas con nivel de educación primaria, y 460 casos menos en personas con nivel de educación secundaria (Figura 4).

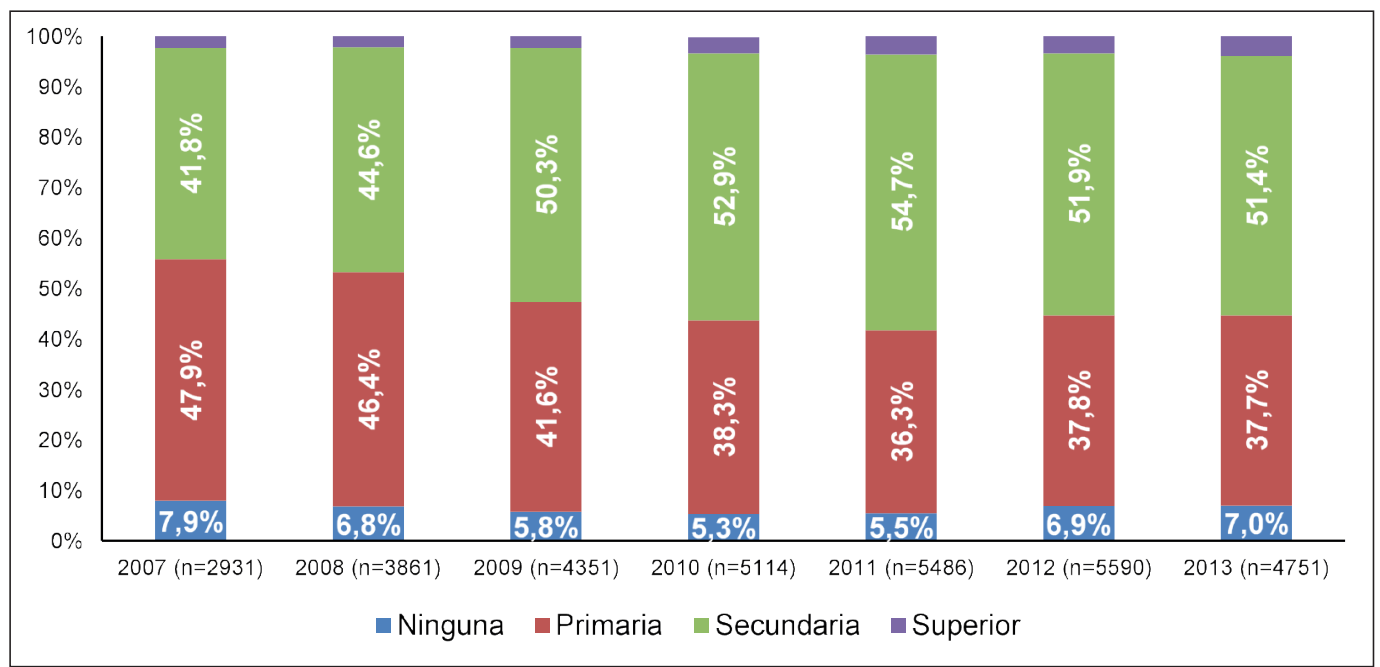

Figura 4. Distribución de intento de suicidio con sustancia química de acuerdo a nivel de escolaridad, Colombia 2007-2013. Fuente: Elaboración de las autoras con datos SIVIGILA 2007-2013.

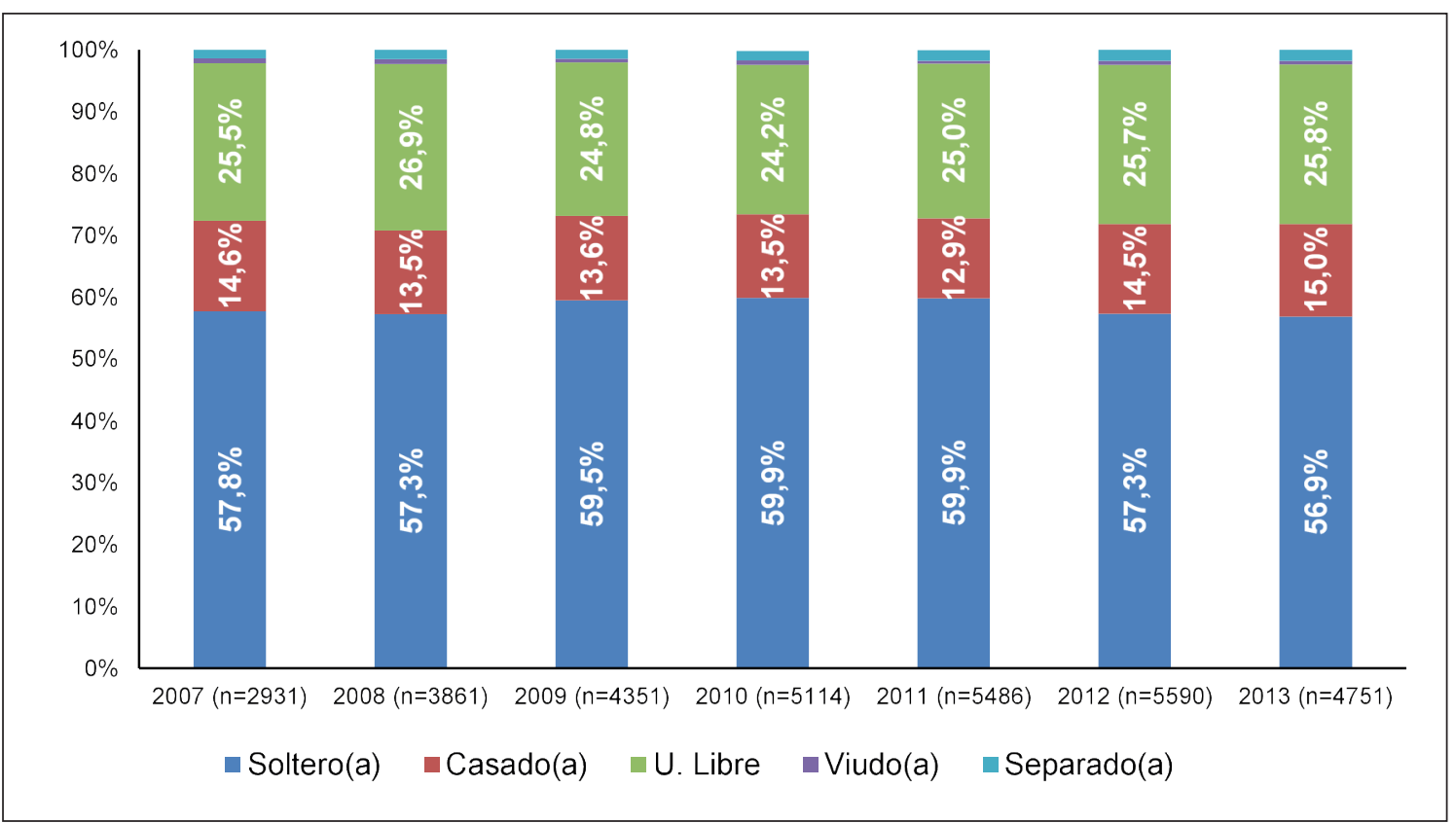

Figura 5. Distribución de intento de suicidio con sustancias químicas, de acuerdo a estado civil, Colombia 2007-2013. Fuente: Elaboración de las autoras con datos SIVIGILA 2007-2013 
En relación a la distribución de casos de intento de suicidio por estado civil, se observa que desde el 2007 hasta el 2013 el grupo de solteros es el que lidera el número de intentos de suicidio en comparación con los demás grupos (58\%), seguido por quienes conviven en unión libre con el $25 \%$ del total de casos. El estado civil con menor proporción de casos es el de los viudos $(0,6 \%)$ (Figura 5).

\section{DISCUSIÓN}

En este estudio se han analizado las características de los intentos de suicidio por intoxicación con sustancias químicas desde el año 2007 hasta el año 2013, encontrando que el comportamiento del evento es similar a lo registrado en la literatura en relación a la mayor ocurrencia en mujeres que en hombres: se documenta que las tasas o proporciones de comportamiento suicida no letal son tres veces mayores en las mujeres que en los hombres ${ }^{15}$. Esta dinámica de intento de suicidio se diferencia del suicidio consumado en tanto que los roles se invierten (por cada cinco hombres que se suicidan en Colombia, sólo una mujer lo hace) pero se explica porque los hombres generalmente buscan métodos más severos y letales que las mujeres, observándose esto no sólo en los suicidios de adultos, sino también en niños y adolescentes ${ }^{16}$.

Llama la atención cómo los jóvenes de 15 a 24 años fueron los más afectados durante el periodo de tiempo estudiado, dejando entrever los retos que tienen las autoridades en salud pública para detectar a tiempo las vulnerabilidades y las susceptibilidades y generar intervenciones oportunas reconociendo que esta es una tendencia global en la cual los jóvenes son uno de los grupos más afectados por este evento. Tampoco se debe desconocer a los adultos mayores, que en su mayoría buscan métodos más letales que los conducen al suicidio consumado ${ }^{17}$. Al respecto, se ha encontrado que algunas de estas susceptibilidades están relacionadas con eventos perturbadores en la infancia tales como la separación de los padres cuando los hombres se encuentran en edades entre los cero a los cuatro años y entre 14 a los 18 años, y las mujeres entre los cero a los 18 años. También se ha documentado que los hijos de padres que usan drogas, abusan del alcohol y presentan conducta suicida, tienen mayor probabilidad de presentar ideación y conducta suicida. De hecho, una gran proporción de las personas que cometen este acto presentan comorbilidad con enfermedad mental en un $70 \%$ depresión ${ }^{18}$, y otros estudios incluso identifican que el género se asocia con trastornos psiquiátricos subyacentes.
Aunque este estudio sólo hizo análisis de los datos de intento de suicidio por intoxicación con sustancias químicas, lo cual no permite hacer generalización del comportamiento del evento a otros mecanismos, es importante reconocer que este es el método más común de intento de suicidio en todo el mundo, y que de esta manera se está aportando información valiosa para identificar las características del evento y movilizar cambios preventivos en todos los ámbitos, como por ejemplo en la normatividad y comercialización de sustancias químicas usadas para el uso cotidiano de la labor agrícola. Reconocer que una parte de quienes realizan actos de autolesión no buscan ayuda médica y permanecen sin ser atendidos por especialistas (subregistro), es el primer paso a considerar en la toma de decisiones para intervenciones comunitarias.

Otro elemento preponderante a tener en cuenta, y que genera gran preocupación en la comunidad académica, es la falta de seguimiento y cuidado posterior al intento de suicidio ${ }^{19}$. La relevancia de captar el caso y brindarle asistencia en salud a la persona está dada porque los intentos de suicidio que preceden el suicidio (una historia de intentos previos de suicidio) son el factor de riesgo más importante al predecir nuevas ocurrencias de conducta suicida; de ahí que el conocimiento de las características y factores asociados con este método sean necesarios para la prevención ${ }^{20}$.

El fácil acceso a sustancias químicas como plaguicidas, rodenticidas e insecticidas deja al alcance de los adolescentes, jóvenes y adultos la disponibilidad de estas sustancias tóxicas para infringirse daño, sin que exista un mayor control de las mismas. Esto representa un riesgo enorme, toda vez que estudios han encontrado que la mayor exposición a organofosforados se ha relacionado con trastornos neuropsicológicos como la depresión y la ansiedad. En el mismo sentido, otros autores identificaron altas tasas de suicidio en las poblaciones agrícolas y una asociación entre la exposición a organofosforados aguda y crónica y los trastornos afectivos, concluyendo que los plaguicidas organofosforados pueden ser parte de la vía causal que conduce al suicidio ${ }^{3}$.

Esto se fundamenta en que, con respecto a los efectos de la exposición a largo plazo al insecticida clorpirifos, la Organización Panamericana de la Salud (OPS) ha indicado que se encuentran involucrados en el sistema serotoninérgico que presenta los cambios a largo plazo en los comportamientos emocionales relacionados con la serotonina ${ }^{17,21}$. En efecto, si bien algunos estudios 
remarcan esta posible asociación, es necesario reconocer que los trabajadores agrícolas a menudo son personas vulnerables que en la mayoría de los casos viven en condiciones de pobreza, lo que los hace susceptibles a una combinación de factores que aumentan el riesgo de bajo nivel educativo, bajos ingresos, consumo elevado de alcohol y enfermedad mental2 ${ }^{22}$.

Si bien los hallazgos de este estudio revelan que la mayoría de los casos se presentaron, entre los años estudiados, en la zona urbana, donde los organofosforados no se usan necesariamente para labor agrícola, es importante destacar que la labor agrícola genera riesgo de mayor índice de depresión e impulsividad.

En cuanto a la pertenencia étnica, se identifica con preocupación cómo los pueblos indígenas presentan una alta ocurrencia del evento, como lo confirman informes recientes de medicina legal. Un elevado número de suicidios consumados se ha presentado recientemente entre estos grupos, atribuyéndose por lo general este hecho a la pérdida de territorios, de identidad y de autodeterminación. Pese a que según el Boletín epidemiológico "suicidio en indígenas", en su informe 2010-2014, hay una tendencia al decremento, este estudio identifica que hay una tendencia al ascenso.

En lo correspondiente a los datos de escolaridad, estos se mantienen muy similares a los hallados en la literatura en los que los intentos de suicidio se concentran en personas con nivel de escolaridad primaria y secundaria, y en menor proporción en personas con estudios universitarios. A pesar de lo anterior, esta no es una variable que deba observarse de forma aislada sino que, por el contrario, debe visualizarse de forma intersectorial, dado que está muy relacionada con las condiciones de pobreza y las necesidades básicas insatisfechas.

En cuanto al estado civil, se identificó que los solteros son los más afectados por este evento, encontrando algunas coincidencias con la literatura; sin embargo, esto varía de acuerdo a la región y a la cultura presentándose, en algunas, mayor ocurrencia en casados.

En términos generales, estos datos permiten hacer una aproximación al comportamiento del intento de suicidio por intoxicación con sustancias químicas en Colombia durante el periodo de tiempo estudiado (2007-2013) pese a que dejan por fuera los otros medios usados para autoinflingirse daño. El país aún no cuenta con un sistema de vigilancia exclusivo de conducta suicida donde se puedan revisar los registros por todas las modalidades, dejando fisuras en el conocimiento de la magnitud del evento en cuanto al uso de armas de fuego, ahogamientos, cortes, saltos al vacío, entre otros.

Si bien es cierto que el sistema de vigilancia SIVIGILA se ha venido robusteciendo y mejorando la calidad de los datos que ofrece año tras año después de su reglamentación (2006), no se puede obviar que en Colombia, como en muchas partes del mundo, este tipo de eventos no tienden a notificarse con frecuencia dado el estigma que generan. Por lo anterior, es necesario tener en cuenta el posible nivel de subregistro que se puede presentar teniendo en cuenta que los casos notificados son sólo aquellos que requieren hospitalización, existiendo situaciones o sucesos que no requieren atención médica dado que los síntomas son leves y son, por lo tanto, tratados en casa.

En conclusión, se encontró una importante incidencia de intentos de suicidio con sustancias químicas que se analizó teniendo en cuenta las características socioculturales de las regiones y los grupos poblacionales que componen la nación colombiana

Se deben generar estrategias de prevención y detección temprana del riesgo de suicidio en la comunidad en tanto que son los jóvenes entre los 15 y los 24 años los más afectados por esta conducta. Es necesario vigilar los intentos de suicidio por todos los mecanismos ya que se desconoce la magnitud del evento y se requiere visibilizar más esta problemática de salud pública en la cual subyace generalmente una enfermedad mental de base no identificada.

Así mismo, se requiere generar espacios de trabajo intersectorial para establecer metas programáticas y objetivos unificados en la implementación de estrategias integrales de prevención reconociendo las particularidades del contexto local.

Por otro lado, resultaría de gran importancia desarrollar estrategias de prevención desde las instituciones educativas (padres de familia, estudiantes y docentes) que promuevan una mirada positiva y promuevan competencias y habilidades para la vida.

De la misma manera, se hace necesario crear un sistema de vigilancia de los intentos de suicidio por todos los mecanismos a nivel nacional, que ofrezca información confiable y valida mediante la cual se pueda visualizar la magnitud del evento y sus características, para que esta clase de hechos no se sigan por la carencia de sistemas de información y vigilancia. Al respecto, existen marcos 
conceptuales que proporcionan orientación sobre esta recomendación ${ }^{23-25}$. La exitosa implementación de modelos que han resultado exitosos, y que son adaptables al medio local, podría mejorar el desarrollo de un programa nacional coordinado de la vigilancia de suicidio mortal y no mortal. Tal coordinación facilitaría la acción basada en la evidencia para reducir la incidencia de suicidio y el comportamiento suicida en todas las poblaciones ${ }^{26}$.

También es necesario reglamentar, en el sistema de salud, la obligatoriedad de las EAPB en brindar un proceso de seguimiento y acompañamiento psicosocial a los adolescentes, jóvenes y adultos, y a sus familias, con un intento no letal durante al menos los primeros seis meses después de la atención inicial, y fortalecer el recurso humano de primer nivel de atención con entrenamiento y capacitación para la detección temprana de enfermedad mental, principalmente depresión.

Finalmente, se debe insistir respecto a la movilización de los actores de cambio y autoridades nacionales a reconocer la conducta suicida como un problema de salud pública y a darle prioridad dentro de la agenda pública, así como a promover estrategias comunitarias que ayuden a reconocer el problema público que más afecta a los adolescentes y jóvenes de manera que se puedan generar intervenciones específicas para estos grupos de edad en condiciones de vulnerabilidad.

\section{DECLARACIÓN SOBRE CONFLICTO DE INTERESES}

Los autores manifiestan que son independientes con respecto a las instituciones de apoyo, y que durante la ejecución del trabajo o la redacción del manuscrito no han incidido intereses o valores distintos a los que usualmente tiene la investigación.

\section{REFERENCIAS BIBLIOGRÁFICAS}

1. World Health Organization. Preventing suicide: A global imperative. Geneve; 2014. [Internet] [Consultado 15 de febrero de 2016]. Disponible en: http://apps.who. int/iris/bitstre0665/131056/1/9789241564779_eng. pdf?ua $=1$

2. Cifuentes, S. Comportamiento del suicidio, Colombia. Instituto Nacional de Medicina Legal y Ciencias Forenses. [Internet]. Forensis. 2012 [consultado 16 de sep 2015]. Disponible en: http://www.medicinalegal. gov.co/documents/10180/188820/FORENSIS + 2013 + 3+ suicidio.pdf/65a683b4-38b2-46a4-b32a-f2a0884b25bf
3. Freire $\mathrm{C}$, Koifman S. Pesticides, depression and suicide: A systematic review of the epidemiological evidence. International Journal of Hygiene and Environmental Health. 2013; 216(4): 445-460.

4. Torres Y, Posada J, Mejía R, Bareño J, Sierra G, Montoya $\mathrm{L}$, et al. Primer estudio poblacional de salud mental Medellín, 2011-2012. [Internet]. Medellín: Alcaldía de Medellín. 2012 [Consultado 17 de sep 2015]. Disponible en: http://www.odc.gov.co/Portals/1/ publicaciones/pdf/consumo/estudios/locales/ C0031052011-primer-estudio-poblacional-de-saludmental-medellin,-2011---2012.pdf

5. Hawton K., Harriss L, Hall S, Simkin S, Bale E, Bond A. Deliberate self-harm in Oxford, 1990-2000: A time of change in patient characteristics. Psychological Medicine. 2003; 33(6): 987-995.

6. Lim M, Lee S, Park J. Difference in suicide methods used between suicide attempters and suicide completers. International journal of mental health systems. 2014; 8(1): 1 .

7. Eddleston M, Phillips MR. Self poisoning with pesticides. British Medical Journal 2004; 328: 42-4.

8. INS. Boletín Epidemiológico Semanal. [Internet]. Semana epidemiológica número 52 de 2015. Págs. 59-61. Disponible en: http://www.ins.gov.co/boletinepidemiologico/Boletn \% 20Epidemiolgico/2015\% 20 Boletin \%20epidemiologico\%20Semana \%2052.pdf

9. INS. Protocolo de Vigilancia en Salud Pública: INTENTO DE SUICIDIO. Versión 01. 2016. Disponible en: http:// www.ins.gov.co/lineas-de-accion/SubdireccionVigilancia/sivigila/Protocolos \% 20SIVIGILA/PRO \% 20 Intento\%20de\%20suicidio.pdf Fecha de acceso: Julio de 2016

10. Tello J. Comportamiento del suicidio en Colombia, 2010. Forensis. 2010; 12:212-34.

11. Hawton K., Van H. Suicide. Lancet. 2009; 373 (9672): 1372-81.

12. Beautrais A, Collings S, Ehrhardt P, Henare K. Suicide prevention: A review of evidence of risk and protective factors, and points of effective intervention. Ed 1. New Zeland: Ministry of Health Wellington New Zeland; 2005.

13. Domínguez L. Caracterización epidemiológica de las intoxicaciones por plaguicidas en Colombia 2011-2012. INS. Informe quincenal epidemiológico nacional. 2013; 18 (20): 243-54.

14. INS. Protocolo de Vigilancia en Salud Pública: intoxicaciones por sustancias químicas. Versión 01 . 2014. Disponible en: http://www.ins.gov.co/lineas-deaccion/Subdireccion-Vigilancia/sivigila/Protocolos $\% 20$ SIVIGILA/PRO \%20Intoxicaciones.pdf

15. Aichberger M, Montesinos H, Bromand Z, Yesil R, Temun S, Rapp A, et al. Suicide attempt rates and intervention effects in women of Turkish origin in Berlin. European Psychiatry. 2015; 30(4): 480-5. 
16. Parellada M, Saiz P, Moreno D, Vidal J, Llorente C, García $P$, et al. Is attempted suicide different in adolescent and adults? Psychiatry Research, 2008; 157(13):131-7.

17. Chen W, Yaun L, Xue R, Li Y, Su R, Zhang Y, et al. Repeated exposure to chlorpyrifos alters the performance of adolescent male rats in animal models of depression and anxiety. NeuroToxicology. 2011; 32 (4): 355-61.

18. Lin C, Yean T, Juang Y, Leong $\mathrm{W}$, Hung $\mathrm{H}$, Ku C, et al. Comorbid psychiatric diagnoses in suicide attempt by charcoal burning: a 10-year study a general hospital in Taiwan. General Hospital Psychiatry, 2012; 34(5): 552-6.

19. Pérez J, Pérez T, Azcuy M, Mirabl G. Intento suicida en adolescentes, un problema de salud en la comunidad. Revista Cubana de Investigaciones Biomédicas. 2014; 33(1): 70-80.

20. Aja Eslava L. ¿Qué ha pasado con el suicidio en Colombia en los últimos 13 años? Forensis. 2009; 11(1):389-98.

21. Wesseling C, Van Wendel B, Keifer M, London L, Mergler D, Stallones L. Symptoms of psychological distress and suicidal ideation among banana workers with a history of poisoning by organophosphate or n-methyl carbamate pesticides. Occup Environ Med. 2010; 67(11):778-84.

22. Meyer R, Salzman C, Youngstrom E, Clayton P, Goodwin F, Mann J. Suicidality and Risk of Suicide: Definition, Drug Safety Concerns, and a Necessary Target for Drug Development: A Consensus Statement. The Journal of clinical psychiatry. 2010; 71(8): e1-e21.
23. Holder $Y$, Peden M, Krug E. Injury surveillance guidelines. Geneva: WHO, 2001. [Consultado Febrero 14 de 2016]. Disponible en: whqlibdoc.who.int/ publications/2001/9241591331. pdf.

24. Matarazzo B, Clemans T, Silverman M, Brenner L. The self-directed violence classification system and the Columbia classification algorithm for suicide assessment: a crosswalk. Suicide Life Threat Behav. 2013; 43(3):235-49.

25. State and Territorial Injury Prevention Directors' Association. Consensus recommendations for injury surveillance in state health departments. Marietta GA: State and Territorial Injury Prevention Directors' Association, 2007. Citado Febrero 28 de 2016. Disponible en: https: $/ /$ www.google.com.co/url?sa $=t \& r c t=j \& q=\&$ esrc $=s \&$ source $=$ web\&cd $=1 \& c a d=$ rja\&uact $=8 \&$ ved $=$ OahUKEwjM46H6tfjLAhUJmh4KHQBGAyQQFggbMAA\& url $=$ https $\% 3 \mathrm{~A} \% 2 \mathrm{~F} \% 2$ Fibis.health.state.nm.us $\% 2 \mathrm{Fdoc}$ s\%2FQuery \%2FSTIPDAConcensusRecommendations. pdf\&usg $=$ AFQjCNE0QkF4-CvPlCvALMKM-nHkxs_Z0Q

26. Data and Surveillance Task Force of the National Action Alliance for Suicide Prevention. Improving National Data Systems for Surveillance of Suicide-Related Events American Journal of Preventive Medicine. 2014; 47(3S2): S122-S129.

Para citar este artículo: Calderón-Ramírez V, Alcocer-Olaciregui A, Vargas-Moranth R. Intentos de suicidio por intoxicación con sustancias químicas en Colombia. 2007 - 2013. Duazary. 2017 julio; 14 (2): 149 - 159. Doi: http://dx.doi.org/10.21676/2389783X.1963 